\section{SLOUGHING SORE-THROAT.}

Under the care of H. A. Pitman, M.D.

[From Notes by G. G. RoGERS, M.D., Medical Registrar.]

EDWARD F., aged 29, was admitted into St. George's Hospital, under Dr. Pitman's care, on June 28th. It appeared that he worked in a saddler's shop, near Leicester Square; and that he was well until about ten days before admission, when he began to feel languid, and had rigors, followed by sickness and loss of appetite. Twenty-four hours later, he experienced pain in the throat, especially on the right side; and this uneasiness grew worse and worse, and interfered with deglutition. However, he had neglected himself until the day of admission, when he came to the Hospital in a very weak condition, with a foul tongue, a feeble pulse, and extremely fœtid breath. He was also very apprehensive about his condition; and this depression of spirits was a marked symptom during the whole of his illness. On admission, a large slough was seen on the right tonsil; and the left side of the throat was also in a very foul condition. The back part of the tongue and fauces were dark coloured. Bark and dilute hydrochloric acid were given every four hours, and he used the gargarisma æruginis frequently. The bowels were rather constipated. On the following day, there were the same appearances about the throat. Deglutition was performed with great difficulty, and the act of swallowing saliva caused almost as much discomfort as taking food. Sesquichlorate of iron and chlorate of potash, with calumba, were given, and eight ounces of port wine ordered. On the 30 th, he was more depressed, and complained of headache, which was relieved by free purgation. There was no particular change in the throat on July 1st, except that the right tonsil appeared a trifle cleaner. Its appearance was again more favourable on the 2nd; but on July 3 rd he was still lower, the breath more offensive, the dysphagia greater, and the throat darker in colour. His spirits were even more depressed than on the two previous days. In the afternoon he sank.

On post mortem examination, forty-four hours after death, the body was found in good condition. There was considerable discoloration of the upper part of the pharynx, as though from decomposition. The papillæ at the base of the tongue were enlarged, and the glands beneath the mucous membrane covering the base of the tongue were extremely large and prominent. There was a large deep sloughy ulcer on the right tonsil; and a depression also existed on the left tonsil, but without any slough or ulceration, more resembling the impression left by a healed ulcer. The substance of the gland on both sides looked healthy. There was no exudation on the mucous membrane. The larynx, œsophagus, and stomach, were healthy. The blood was universally fluid, except some semi-clotted blood in the heart. The viscera presented no appearances worthy of note.

Remaris. We give the details of another case of the epidemic sore-throat which is now extensively prevalent in some parts of the metropolis, and which bears certainly a great affinity to diphtheritis, if it be not another form of the same affection. We shall not, however, offer any remarks on this at the present time, as we hope next weck to lay before our readers the case of the servant to the family from which a fatal case was reported in our last number, and which promises to terminate more fortunately than those did. As the nature and treatment of this disease are matters of great interest at present, we shall be careful to report all of these cases of which we can find full details.

\section{BURN FOLLOWED BY TETANIC SYMPTOMS IN AN} INFANT.

Under the care of H. C. Johnson, Esq.

We append short notes of a case of burn followed by tetanic symptoms in an infant, principally as a companion to the cases of traumatic tetanus in infants alrealy reported in this JoURsal, and also to show how large a quantity of stimulants may be given in cases of such extreme prostration even at a very early age.

Thomas M., aged 15 months, was admitted on June 9th, under the care of Mr. H. C. Johrison, on account of a very severe burn received two days before. The burn involved the skin of the legs, thighs, scrotum, and nates, and was sloughing in many places. On the afternoon of the tenth day on which it was brouyht in, the child became extremely pallid, and was almost dying. It had refused the breast during the last day or two. Four ounces of port wine were given, and the child rallied. Next day the state of weakness was so great that it was found necessary to give eight ounces of wine. This did not accelerate the pulse beyond the normal standard. Occasionally a drop of laudanum was given, and milk was ordered, besides the breast, which the child now took again. For the next eight days, it went on taking the same quantity (eight or nine ounces) daily. During all this time, each day appeared to be certainly the last. At times, for hours together, the head and neck were in rigid spasms of opisthotonos, and the arms thrown out in similar spasms. Still it would suck, and took wine and milk freely.

It died on June 19th. There was no post mortem examination.

\section{OHriginal Communitations.}

\section{THE APPLICATION OF CARBONIC ACID GAS TO THE INTERIOR OF THE BLADDER.}

By T. SkInNer, M.D., Liverpool.

Is the Jorrnnar for June 12th, I perceive that both Dr. Johns and myself have been forestalled in our claims to priority. I willingly resign the contest for priority, but unhesitatingly lay claim to originality of observation. Without further comment, I proceed to the subject of my paper.

In my letter in the Journal of May 29th, I promised "to describe a much safer, simpler, more portable, and more effectual apparatus", for the local application of carbonic acid gas to the interior of the bladder, than that used by Dr. Johns. I also said that I would endeavour to disabuse the mind of the faculty in regard to the absurd idea of poisonous, disagreeable, or dangerous results; and that I would give a digest of $m y$ own experience, with a few of my most interesting cases.

I will first describe my apparatus, adding a few remarks on its use and mode of administration.

r. Description of Apparatus. The accompanying diagram,

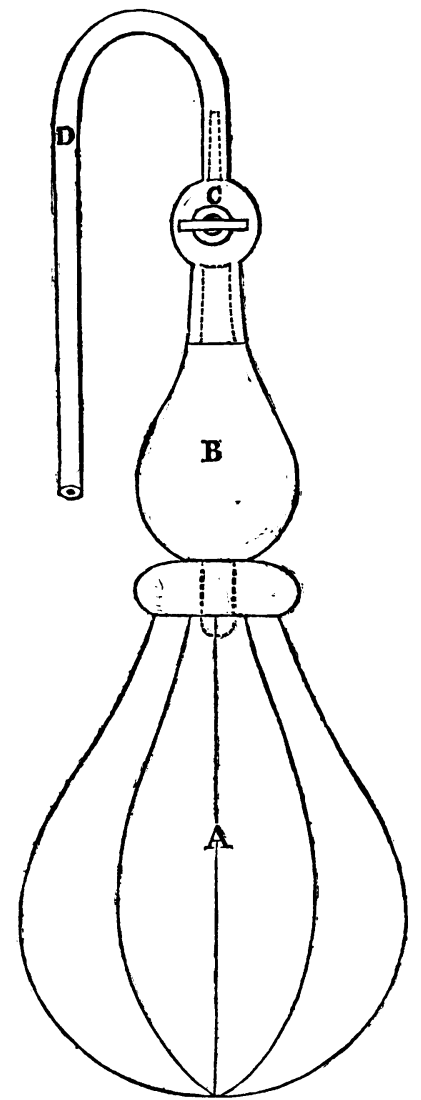

奂

about one-fourth of the proper size, will aid my description. The apparatus will be observed to be composed of four separate pieces, A, B, C, and D. A is a caoutchouc bottle, capable of containing four fluidounces, covered with green varnish (which I prefer, as being more durable and elastic). B is a glass buli, 
made from a pretty strong tube, with two male sockets, fitting into $\mathrm{C}$ and $\mathrm{A}$. This glass bulb $\mathrm{B}$ is intended to contain a long strip of sponge, to be saturated more or less with chloroform when wanted. The sockets must be filed or roughened, and the cement for the extremities at $\mathrm{A}$ and $\mathrm{c}$ must be of plaster of Paris, or of such a material as will not be acted upon by the vapour of chloroform, as gutta percha, shellac, and most of the gum resins, are. The cork at the A extremity must be attached firmly to B, and fit easily yet closely into A. C is of brass-a simple stopcock, with a male and female socket; the female socket receives $B$ with plaster of Paris; the male socket passes tightly into $\mathrm{D}$, with varnish, or any other, or no cement. $\mathrm{D}$ is a gum-elastic catheter, No. 8, perforated, not at the side, but at the distal extremity, six inches long. B and c may both be made of a single piece of metal, and should be made proportioned to $\mathrm{A}$ and $\mathrm{D}$. I may here add, that $\mathrm{B}$, as a reservoir for chloroform, has been added since I saw Dr. Johns' paper The only other requisites are-1. A small glass funnel, which may be procured at any chemist's shop, two inches in diameter at the rim, and fitting easily into A without a cork, the diameter of the socket in a being exactly half an inch. 2. A clear glass bottle, with a wide neck, capable of holding about six or eight ounces of liquid, with an India-rubber stopper and flexible tube. 3. Some carbonate of soda and tartaric acid, in crystals or otherwise, or in separate powders of one drachm each. Lastly, for my own use, I have two supplementary parts; viz., gum-elastic catheters of various sizes, and full length for males, fitting on to $\mathrm{C}$; and a second edition of $\mathrm{B}$, made of wood, without the reservoir for chloroform.

II. Use and Mode of Administration. To fill the elastic bottle there are two methods. It may be easily done by at taching it to a gasometer, previously exhausting it by pressure but there are few of us possessed of such cumbrous and expensive apparatus, or likely to become so. The only other and the simplest means is as follows:-Disunite $\mathbf{A}$ and $B$ and insert the glass funnel into $A$; fill $A$, funnel and all, brimful of water; then make a pneumatic jar of it, and invert it under water. Put one drachm of carbonate of soda into the widemouthed bottle, with half an ounce of water; add to it a drachm, or nearly so, of tartaric acid, in small or bruised crystals; quickly put on the stopper with the tube; wait a fer seconds, to allow the atmospheric air to be expelled from the bottle and tube, which may readily be ascertained by placing the tube in the mouth, the curbonic acid having a peculiar, slightly acid taste; then pass the tube beneath the funnel. The gas passes up into the elastic bottle, and the water descends to its own level in the basin. When filled, turn the stopcock $\mathrm{C}$ as shut; take the caoutchouc bottle off the extremity of the funnel, and connect it with $\mathrm{B}$. There need be no incon. venient haste about the latter part of the process, as carbonic acid is a heavy gas, and not rapidly diffusible through a narrow neck.

The apparatus is now charged, and ready for use. If it is well made, and the administrator understands the manipulation of gases, the gas remains pure for many hours after being charged. I have often tried it five hours afterwards, by means of lime-water, which it rapidly renders white and milky. If chloroform is wanted to be injected along with the carbonic acid, ten to thirty minims may be dropped on the sponge in the reservoir $\mathrm{B}$, before uniting it to A. For a pneumatic trough zuse a washhand basin, and one of Griffin's little beehive stands for supporting a pneumatic jar; but this latter is not absolutely necessary. I am sorry I cannot say where the apparatus may be had; as, for want of ingenious workmen here, I had to cobble my own up myself. I have been thus minute in details, in order that intelligent instrument-makers may be enabled to make it elsewhere.

In regard to the mode of administration, the patient may lie on the side or back. I prefer the left side, as in most obstetric operations. Without the slightest exposure, and, if there is no hyperæsthesia or morbid sensibility of the parts, without the slightest pain, $D$ is introduced into the bladder, the viscus having been previously emptied, either by the patient herself, or by the catheter. The stopcock is then turned open; the elastic bottle steadily, and not too rapidly, compressed until emptied. The bottle is kept compressed, and the whole apparatus is withdrawn from the bladder, leaving the gas locked up in that viscus. In some cases, it is necessary to dilute it with atmospheric air; this may be done with an ordinary wineglass, or similar vessel. Equal parts or any proportion of the gas may thus be injected as desired; the proportion of atmospheric air being first iutroduced, and the remaining space in the bottle being filled up by the carbonic acid.

If a current of the gas is thought necessary (which I do not believe it to be), the gas may be allowed to return to the bottle, and be re-injected as often as necessary, before withdrawing the instrument. It will still be found to be unaltered carbonic acid.

If at any time a feeling of lethargy or drowsiness comes over the patient, which I have seen once, she ought to expel the gas, or have it drawn off with a catheter. Patients can generally expel it themselves at any period. At the next application, it should be diluted.

It may be applied as often as three times a day. I have repeatedly used it twice a day; sometimes once a day, and once in the two or three days, according to circumstances and the nature of the case.

I have not in general found it or thought it necessary to wash out the bladder previously; but, in cases accompanied with much discharge of mucus or blood, this step is decidedly a desideratum. In washing out the bladder, the expense of a double silver catheter is unnecessary. My own instrument is quite adequate to the purpose. By introducing $D$ into the bladder with A, filled with tepid water, the bladder is injected; $D$ is disconnected from $\mathrm{C}$; and the contents of the viscus are allowed to escape. This may be repeated as often as necessary, and without withdrawing $D$ at all; it may be reconnected with A, and charged with carbonic acid; and the injection made as directed. This is not so convenient with the instrument, as it interferes with the charge of carbonic acid, which I invariably put in at home, and carry to a case. I prefer using the ordinary flexible catheter, No. 8; and a two-ounce male glass syringe, or any other with which the same process may be carried out. The double catheter, however, is better adapted for this purpose.

Chlorine and other gases have been injected by me with the same instrument, variously diluted; but it is only lately that I have done so. I would require to make more observations, and have greater opportunities of making them than I at present enjoy, before giving them to the public. Chlorine, so far as I have tried it, promises fair in chronic catarrh and inflammatory affections of the bladder. I have only tried it in one such case, much diluted with common air, and without allowing it to remain, with decided benefit. I feel confident that, with great care, much might be done to improve the therapeutics of the urinary bladder by such and other appliances.

Although it has taken me some time to explain my in. strument and method, I can confidently assert that the charging of the apparatus does not occupy five minutes; and the operation of injection need not occupy one-half as much, unless the bladder require to be washed out.

[To be continued.]

Action for Recovery of Fees, (Sittings in Nisi Prius, at Guildhall, before Mr. Justice Erle and a Special Jury.) Lee $v$. Candler, administrator. Mr. M. Smith and Mr. Kars. lake were counsel for the plaintiff; and Mr. Huddleston and Mr. J. Denman for the defendant. This was an action brought by Dr. Edwin Lee against the defendant, who was the adminis. trator of Mr. Halstone Browne, to recover 150 guineas for his attendance on Mr. Browne. It appeared that Mr. Browne was at Hyères, in a very delicate state of health, in 1856. The plaintiff was in the habit of spending the winter in the south of France, and happened to be at Hyères in 1856. Mr. Browne placed himself in the hands of Dr. Lee. In the course of the month of April 1857, Mr. Browne was desirous of returning to England, and he wished Dr. Lee to accompany him. Dr. Lee stated to him that he was under the necessity of attending the remains of a young lady to England, but he could go part of the way with him. They went to Lyons, where Dr. Lee left Mr. Browne in the charge of a medical friend. Dr. Lee proceeded with the remains of the young lady to England, and in a few days returned to Paris, where he received a telegram informing him that Mr. Browne was very ill. Dr. Lee then hastened to Lyons; but when he arrived there he found that Mr. Browne was dead. The plaintiff then made a claim for his medical attendance, his journey to Lyons and back; and this he estimated at 150 guineas. Mr. Justice Erle left it to the jury to say whether, in their opinion, Dr. Lee attended and was employed as physician; and should also ask the jury what amount of remuneration they considered Dr. Lee entitled to. The defence was, that the attendance of the plaintiff' was that of a physician, and therefore he was not entitled to recover. It was then contended that the charge was too great. The jury retired, and, on their return, said the plaintiff was employed as a general practitioner. Verdict for the plaintitf; for tifty guineas. 